\title{
Pioneers in Computing
}

CoffeeART by

Use the clues to find the last names of famous Computer Science pioneers

Amy Whitaker

www.ArtByAmy.gallery

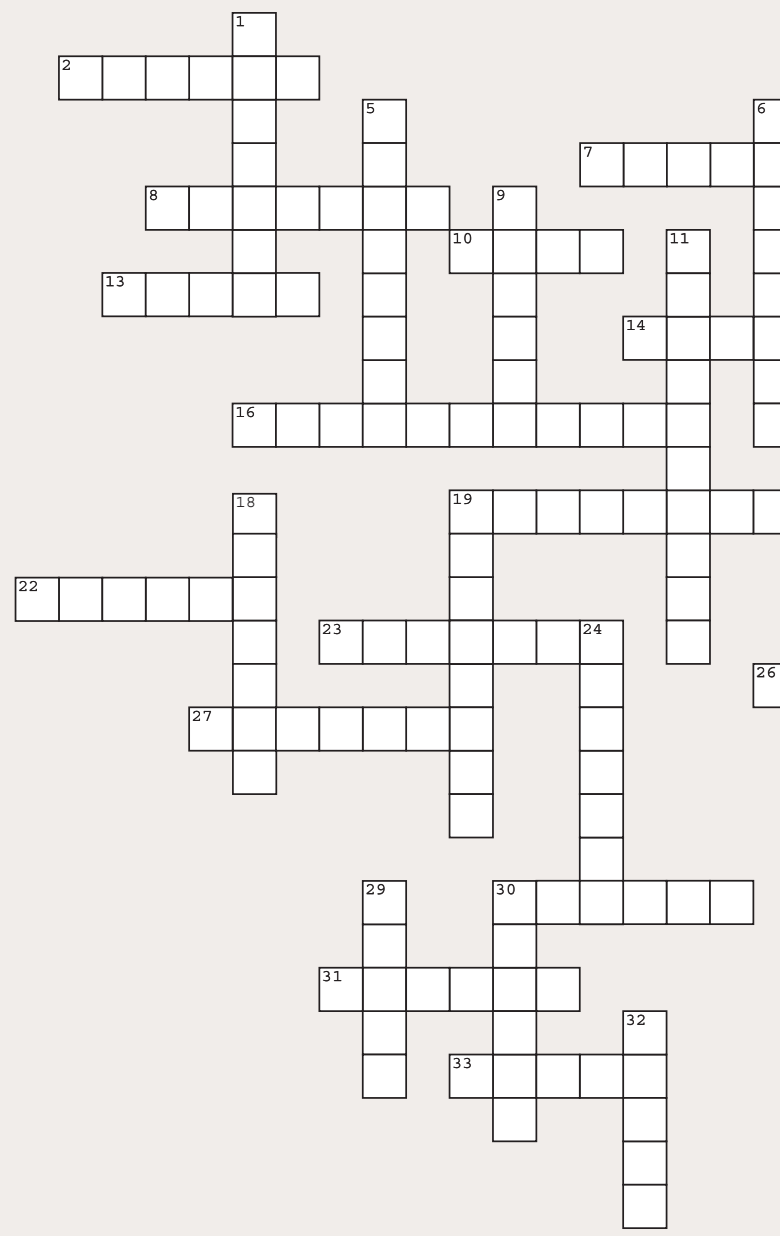

\section{Across}

2 First Turing Award

7 Designed the Acorn Micro-

Computer and the ARM

instruction set

8 Calculated trajectories, launch windows, and emergency backup return paths for NASA

$10 \mathrm{bNf}$

12 Defined the tree-adjoining grammar formalism

13 Seminal work in compilers, code optimization, and parallelization Turing Award winner

14 Computer mouse

16 WWW
19 Ruby, MINASWA

21 and, or, not

$22 \mathrm{COBOL}$

23 SIMULA 67

26 Analytical Engine

27 LATEX

28 Lambda Calculus

30 Pioneer in distributed

computing, co-developer of a famous algorithm with Lamport

31 Father of theoretical computer science

33 Pioneer in data structures; co-author of Fundamentals of Computer Algorithms

\section{Down}

1 Co-creator of $C$ and UNIX; R of K\&R C

3 Semaphores but no GOTOs

4 Co-author of the Dragon Book

5 Co-creator of $C$ and UNIX

6 Co-developer of Smalltalk-80

9 Formula Translation

11 Stored-program architecture named for him

15 Communicating Sequential Processes (CSP) and Quicksort

17 First programmer
18 Invented the spanning-tree protocol (STP)

$19(())$

20 Invented computerized telephone switching

24 Started the GNOME, Mono, and Xamarin projects

25 ENIAC

29 Big $O$ and TeX

30 Started revolution in VLSI with Mead and invented generalized dynamic instruction handling

32 Pascal, Modula-2 and Oberon 